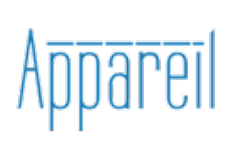

Appareil

3| 2009

Concerts publics et formes de la sensibilité musicale

\title{
Le rituel du concert et la question du sacré
}

\section{Renaud Tarlet}

\section{(2) OpenEdition}

Journals

Édition électronique

URL : http://journals.openedition.org/appareil/841

DOI : 10.4000/appareil.841

ISSN : 2101-0714

\section{Éditeur}

MSH Paris Nord

\section{Référence électronique}

Renaud Tarlet, « Le rituel du concert et la question du sacré », Appareil [En ligne], 3 | 2009, mis en ligne le 03 juillet 2009, consulté le 30 juillet 2020. URL : http://journals.openedition.org/appareil/841 ; DOI : https://doi.org/10.4000/appareil.841

Ce document a été généré automatiquement le 30 juillet 2020.

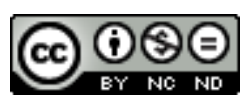

Appareil est mis à disposition selon les termes de la Licence Creative Commons Attribution - Pas d'Utilisation Commerciale - Pas de Modification 4.0 International. 


\title{
Le rituel du concert et la question du sacré
}

\author{
Renaud Tarlet
}

\section{Introduction}

1 Cette intervention dans le cadre du séminaire portant sur la thématique «Concerts publics et formes de la sensibilité musicale » n'est pas, nous tenons en premier lieu à le préciser, celle d'un spécialiste d'une quelconque manière de la sociologie de la musique ou de l'art. Notre apport, s'il existe, sera celui d'un apprenti anthropologue du sacré et consistera à tenter de proposer une «boite à outils » conceptuelle afin d'appréhender le rituel du concert symphonique, voire, plus largement, le rituel du concert de façon plus générale. Le regard empirique employé ici sera tout à fait superficiel, tendant à dégager des généralités que les spécialistes pourront approfondir.

2 Il s'agit pour nous de replacer ce rituel dans le cadre de son contexte social, comme un élément contenant, de manière inconsciente, les structures de la société dans laquelle il se produit. Autrement dit, nous nous proposons d'appréhender le rituel du concert dans le cadre de ce que les anthropologues appellent un système symbolique. En ces lignes, le rituel du concert sera considéré comme un élément contenant les formes fondamentales d'un tout culturel (en ceci qu'il organise un ensemble de représentations) et social (car porteur d'une organisation et d'une structure sociales) plus vaste.

3 Mais au-delà de cette appréhension, nous voudrions tenter de porter un regard articulé autour du concept de sacré, afin de proposer des éléments de réflexion et de débat concernant l'éventuelle parenté du rituel du concert symphonique avec les phénomènes religieux et politiques.

4 Il est à présent temps d'évoquer brièvement les enjeux épistémologiques dont le regard que nous proposons ici est porteur. Le sacré est un concept inventé par Durkheim, qui constate que toutes les sociétés sont organisées autour d'une division entre choses sacrées et profanes. Durkheim définit comme sacrées les choses marquées par 
l'ambivalence, tenues à distance autant que conjurées, révérées autant que craintes, porteuses de tabous, d'interdictions et d'obligations. On pourrait ainsi appliquer ce concept au drapeau français, pour prouver sa sacralité au sens durkheimien. Ce symbole contient l'ambivalence typique des choses sacrées : il est tenu à distance et frappé d'interdits (on ne s'en fera pas des sous-vêtements ou on ne l'utilisera pas pour s'essuyer ou se moucher, par exemple, on ne passera pas ses journées à se promener avec), mais aussi conjuré (on affirme sa présence lors des cérémonies collectives ayant trait à la nation), il est révéré et craint voire honni, parfois par les mêmes personnes (on peut révérer le symbole de la France révolutionnaire et abhorrer celui du colonialisme français, ou l'inverse), porteur enfin d'obligations (dans certaines occasions, on doit le saluer, par exemple).

5 Or Durkheim, dans un effort d'objectivation qui ouvre les faits religieux à l'analyse sociologique et anthropologique, définit les faits religieux comme ceux ayant trait aux choses sacrées. Nous pourrons en conclusion débattre des apports et limites de cette appréhension concernant le concert symphonique et, plus largement, les faits artistiques. Ce sera donc, durant cet exposé, notre question directrice que d'essayer de déterminer s'il y a du sacré dans le concert.

Cependant, poser la question de la sacralité du concert contient implicitement un positionnement critique par rapport au grand courant théorique de l'anthropologie française, qu'il n'est en rien excessif de considérer comme la grande révolution de l'anthropologie du xxe siècle : le structuralisme.

7 Le structuralisme, en anthropologie, a été fondé par Claude Lévi-Strauss. Or, pour les structuralistes, à sa suite, le sacré est une notion secondaire et peu intéressante. Le structuralisme, on le verra plus loin, est une pensée de l'ordre et du classement, pour laquelle l'ambivalence du sacré reste incompréhensible. L'acte sacrificiel, conjonctif (car il cherche le contact avec des forces extra-sociales) puis disjonctif (car une fois effectué, il les renvoie d'où elles viennent) est pour les structuralistes un non-sens. Il est pour eux une sorte de "rustine" symbolique, un artifice inventé par les sociétés humaines pour masquer leur ignorance : le sacré, en quelque sorte, est invoqué face aux phénomènes inexplicables. Pour l'essentiel, Lévi-Strauss pense qu'il ne saurait exister une science du sacré (ce qui est une attaque contre Durkheim) puisque, là, où il y a science, il n'y a plus de sacré. En effet, la science étant amenée à expliquer les phénomènes incompréhensibles, sa progression ne pourrait ainsi que signifier la régression subséquente du sacré, qui ne découlerait que de l'ignorance ou de l'incapacité à expliquer rationnellement des phénomènes.

8 Le structuralisme, cependant, est particulièrement remis en cause, surtout en ce qui concerne son peu d'intérêt concernant le sacré, par la théorie de l'anthropologue René Girard, celle du bouc émissaire, théorie sur laquelle nous reviendrons.

9 Le rituel du concert peut donc être un bon prétexte pour, ensemble, nous livrer à un rapide état des lieux épistémologique de l'anthropologie du sacré et de ses apports. Il nous semble important, de plus, loin de tout terrorisme intellectuel ou de tout esprit de chapelle, de rappeler la possibilité de nous nourrir aux sources et du structuralisme, qui a tant apporté à l'anthropologie, et à celles de ces prédécesseurs ou critiques, en réalisant, à travers l'objet qui est ici le nôtre, un inventaire qui essaiera autant que possible d'être honnête et ouvert, sans rien abandonner en ce qui concerne la nécessaire exigence d'un esprit critique. 
10 Dans un premier lieu, nous proposons donc d'expliciter rapidement la démarche de l'anthropologie structurale, avant d'appliquer une analyse structurale à l'orchestre symphonique et à son public. Cette analyse concernera tant les structures de l'un et de l'autre que les rapports d'échange les mettant en relation.

11 Cependant, il nous semble ensuite indispensable de relever les limites de cette anthropologie structurale pourtant si féconde, en relevant des «anomalies structurales» dans les échanges orchestre-public, avant d'exposer la théorie girardienne du sacré pour tenter d'en appliquer les apports au rituel du concert symphonique.

12 Enfin, nous tenterons de placer ce rituel dans son contexte politique et social, en abordant la problématique de l'indifférenciation. Nous essaierons de démontrer que cette dernière, dans certains cas, touche tant le rituel qui nous intéresse ici que la société dans lequel il prend place.

13 Ce cheminement nous permettra de conclure en nous interrogeant sur les différents régimes du sacré, les différences et points communs entre ses manifestations à travers les époques et les cultures et pour finir les rapports entre les faits artistiques, religieux et politiques.

\section{Une analyse structurale du rituel du concert}

\section{Un petit point à propos du structuralisme}

14 Le structuralisme anthropologique s'est constitué à partir des prémisses de la linguistique structurale. Cette dernière appréhende le langage comme un système inconscient composé de sons élémentaires, les phonèmes, qui en eux-mêmes sont dénués de sens et n'en prennent que par les rapports, particulièrement d'opposition, qu'ils entretiennent entre eux.

15 À la suite de Marcel Mauss, les anthropologues structuralistes vont voir dans les cultures et les systèmes sociaux des systèmes symboliques composés de multiples dimensions, dont les éléments, les symboles, n'ont de sens qu'à travers leurs rapports au sein d'un système inconscient. Le structuralisme ne valorise donc pas l'étude des contenus (des symboles donnés) en premier lieu, mais celle des formes, autrement dit des écarts différentiels qui forment le système symbolique et donnent sens à ses éléments. Prenons un exemple pour clarifier ce propos qui peut sembler un peu obscur.

16 Nous évoquerons ici les observations de Bronislaw Malinowski ${ }^{1}$ qui représentent un classique de l'ethnographie. Certes, Malinowski n'était pas structuraliste (le structuralisme anthropologique n'existait pas encore quand il a écrit Les Argonautes du Pacifique occidental), mais la précision de ses descriptions et la systématicité de sa méthode d'observation nous permettent d'opérer une analyse structurale des sociétés qu'il décrit dans cet ouvrage. Malinowski relève, dans l'archipel des Trobriand qui est un des principaux terrains de son observation dans Les Argonautes, que la structure des villages est toujours à peu près la même. Ils sont organisés autour d'une place centrale, le baku, où se déroulent les cérémonies collectives. On y trouve traditionnellement la maison du chef du village, ainsi que les greniers à ignames, cet aliment ayant un statut particulièrement prestigieux dans la culture trobriandaise. Or, les bâtisses autour du baku sont surélevées et leurs murs laissent entrer la lumière, par opposition avec les 
bâtisses du pourtour, du reste du village, qui sont construites à même le sol et restent sombres, impénétrables au regard extérieur. Le baku est ainsi le lieu des cérémonies collectives et publiques, pour l'essentiel, par opposition au pourtour qui est le lieu de l'intimité et de la vie privée.

On constate donc qu'à une opposition géographique centre/périphérie correspond toute une série d'oppositions afférentes, lumière/obscurité, privé/public, surélevé / à même le sol, Chef / reste de la tribu: ces couples d'opposition forment système, et traversent les différentes dimensions de la société trobriandaise. Ainsi, à une opposition géographique correspondent des oppositions architecturales, esthétiques, de hiérarchie sociale, de pratique rituelle par exemple. Les contenus sont organisés autour d'une structure qui leur fait former système: un écart différentiel, une différence toujours de même ordre. Ainsi, le centre est à la périphérie ce que le prestigieux est au banal, ce que le chef est au reste de la société, ce que le lumineux est au sombre, le haut au bas, et ainsi de suite.

Pour reprendre une métaphore célèbre de Claude Lévi-Strauss, une société serait un ensemble de systèmes symboliques reliés par une structure commune, un peu à la manière d'une partition d'orchestre dans laquelle chaque instrument aurait sa portée, mais en relation avec tous les autres, tous étant accordés et reliés dans l'exécution de l'œuvre considérée dans son intégralité. Tout comme harpe, contrebasse, vents et cuivres dans un orchestre interprètent leur propre partition au sein de l'ensemble, les réalités politique, rituelle, de représentations, de morphologie sociale d'une société donnée appartiendraient, de manière inconsciente, au même système social global.

19 À ce regard sur les sociétés considérées en tant que systèmes inconscients d'oppositions et d'écarts différentiels, l'analyse structurale ajoute une appréhension des rapports sociaux avant tout comme faits d'échange régis par une loi de réciprocité. Une société est ainsi régie selon les structuralistes par des échanges, de femmes, d'objets, de signes, entre autres.

20 C'est donc à cette analyse structurale du rituel du concert symphonique, appréhendé aussi bien en tant que système symbolique porteur d'oppositions et d'écarts différentiels, qu'en tant que pratique donnant lieu à des échanges, que nous allons à présent nous atteler.

\section{Analyse structurale de l'orchestre et du public}

21 Nous allons maintenant, comme dans le cas du village trobriandais, tenter de mettre en évidence les oppositions qui structurent l'orchestre symphonique, pour les replacer dans le cadre d'un système symbolique traversant plusieurs dimensions de la réalité sociale. Nous remarquerons tout d'abord que la disposition des instruments au sein de l'orchestre est régie par une opposition gauche/droite (quand on se place du point de vue du public) qui correspond à une opposition aigu/grave. Ainsi, les instruments les plus aigus sont placés à gauche de l'orchestre, les plus graves à droite. Communément, les cordes sont devant, les vents derrière, les percussions au fond. De plus, les cuivres sont en général placés derrière les bois. Il existe donc une opposition structurante devant/derrière. Nous constatons qu'une opposition spatiale (gauche/droite) correspond à une opposition sonore (aigu/grave).

Le chef d'orchestre et le premier violon solo sont placés sur le devant de l'orchestre, ainsi que le chanteur et/ou la chanteuse solistes. Le premier violon demande le « la » au 
chef d'orchestre, est salué par le chef et le public, commande le lever des musiciens et accueille le chef d'orchestre. Il représente l'orchestre face au chef et au public.

Les oppositions spatiales au sein de l'orchestre sont donc sonores, mais aussi sociales. Premièrement, les positions les plus prestigieuses (chef, premier violon solo, solistes, chanteurs solistes, par exemple) sont placées vers l'avant. De plus, le classement spatial des instruments correspond à un classement en dignité, qui donne une importance et un prestige social différents à chacun d'entre eux. Il est ainsi plus prestigieux de jouer du violon que de l'alto, de jouer d'un bois que d'un cuivre. Dans la même logique, les oppositions de prestige au sein de l'orchestre correspondent à des oppositions dans la hiérarchie sociale. Les positions les plus prestigieuses et les plus gratifiantes sont le plus souvent occupées par des membres des classes sociales les plus favorisées. Ainsi, les joueurs d'instruments à cordes sont-ils bien plus souvent issus des classes favorisées, les cuivres étant des instruments plus démocratiques: on apprend plus souvent à jouer des cuivres dans les harmonies, ce qui permet un recrutement plus populaire.

Ainsi, les instruments placés à gauche et en avant de l'orchestre, aigus, prestigieux, joués par des membres des classes supérieures, s'opposent aux instruments situés à droite et au fond, graves, moins prestigieux, joués plus souvent par des membres des classes moins privilégiées.

Nous remarquons aussi qu'un schème (c'est-à-dire un mode de perception inconscient reposant sur un écart différentiel) opposant l'unique, valorisé, à la multitude, dévalorisée, structure aussi les logiques de prestige au sein de l'orchestre. On peut ainsi être derrière les cordes, comme le piano, mais plus valorisé parce qu'unique dans l'orchestre. Mais, toutes choses égales par ailleurs, l'exceptionnel le plus valorisé est le plus proche du public, semble-t-il.

Les mêmes oppositions systématiques et inconscientes, les mêmes schèmes de perception, opèrent au sein des divisions de l'orchestre pour créer de nouvelles différenciations. Ainsi, les vents sont divisés entre aigus à gauche et graves à droite, bois devant et cuivres derrière.

L'orchestre symphonique représente donc un système symbolique multidimensionnel comparable à celui du village trobriandais que nous exposions plus haut. À des oppositions spatiales correspondent des oppositions sonores, de type d'instrument, de prestige, de recrutement social, par exemple. Nous constatons que toutes ces dimensions sont structurées selon une forme commune, selon un système commun qui repose sur un écart différentiel constant : la gauche est à la droite ce que l'avant est à l'arrière, ce que l'unique est au multiple, ce que le valorisé est au dévalorisé et ainsi de suite.

Nous réalisons donc que l'orchestre représente une topologie sociale, thématique fort sensible dans un film à succès tel que Les choristes. La chorale qui y tient la vedette correspond à une sorte d'utopie où l'ordre politique et social est fantasmé comme découlant de l'ordre naturel. Le plus «naturellement » talentueux chante en soliste, tout le monde obéit au chef qui est le seul à connaître le bien, chacun occupe une place liée à ses possibilités, le plus mauvais tient la partition, enfin le méchant incorrigible, irrattrapable, doit être expulsé du groupe. La chorale des choristes appartient à un système symbolique où elle tient le rôle plus ou moins affirmé de métaphore politique, tentative de retour à un « ordre naturel » fantasmé qui fleure bon le pétainisme... 
29 Nous constatons donc qu'à travers l'orchestre, ce sont un système de représentations et un ordre social qui se donnent à voir sans que la plupart des acteurs du rituel en soient conscients. Mais ces divisions et structures collectives inconscientes sont tout aussi sensibles dans l'organisation et le placement du public du concert symphonique.

30 Suivant la même logique que l'orchestre, le public d'un concert symphonique est différencié, certaines places étant plus valorisées que d'autres, celles du centre étant plus cotées que celles des extrémités (topologie que l'on retrouve dans les classements politiques). Être devant (orchestre) ou en haut (balcon) sont les positions les plus valorisées. Cependant, un principe d'éloignement tempère cette hiérarchie selon laquelle le haut est valorisé. Les places trop en haut sont dévalorisées. Encore une fois, une topologie contient des classements qui reflètent l'ordre social. Bien entendu, les places les plus recherchées sont les plus chères et sont donc réservées de fait aux membres du public appartenant aux catégories sociales les plus favorisées.

\section{Orchestre et société}

31 L'orchestre symphonique et le rituel auquel il participe sont donc porteurs de structures et de classements sociaux et socialement agissants. L'analyse structurale nous ouvre ainsi la porte d'une mise en contexte du rituel du concert par rapport aux représentations et à l'organisation sociales dans lesquelles il s'insère.

En tout cas, la question de savoir s'il n'y aurait pas des éléments en rapport avec la sacralité et le religieux dans le rituel du concert symphonique semble légitimée par l'importance qu'y tient le discours $d u$ «talent». Les positions les plus valorisées sont ainsi censées être distribuées selon un principe métasocial, celui du «don». Le compositeur, le chef d'orchestre ou les solistes sont fantasmatiquement désignés par une nature qui les aurait pourvus des capacités nécessaires, sans l'intervention de la société et des hommes. En réalité, c'est la société qui définit ce qu'est le talent; talent qui, le plus souvent, correspond à une série d'aptitudes largement conditionnées par l'origine et l'expérience sociales. Évidemment, celui qui, dès la petite enfance, baigne dans un univers culturel et musical propice a bien plus de probabilités de devenir un musicien "talentueux » qu'un autre. Les agents sociaux, à travers l'orchestre et la pratique musicale, appréhendent donc une "nature » qui est en fait largement un artefact social (c'est la socialisation dans des milieux sociaux propices qui «fait» largement le «talent naturel» ou «inné »), et cette "nature » qui en réalité est socialement construite, par un retour magique, vient ensuite justifier la hiérarchie sociale. Le chef d'orchestre et les solistes ne sont le plus souvent des membres des classes privilégiées que parce que c'est dans ces classes privilégiées que le «talent »se manifeste le plus souvent (et pour cause).

Il s'est donc développé, avec la montée en puissance de la bourgeoisie, un discours du " génie » dont Mozart est l'un des plus beaux exemples. D'après la légende, il crée ses premières œuvres dès la petite enfance, ce qui semble confirmer la « naturalité » de son «don». La précocité du musicien est donc un signe qu'il faut guetter comme la confirmation de sa désignation par un au-delà de la société : son "talent » apparaît avant même sa socialisation, il ne saurait être le fruit d'une éducation ou d'une intervention humaine.

34 Le principe justificatif de l'ordre social qui apparaît dans la pratique musicale ou artistique est en parfaite homologie avec les discours du même ordre ayant cours dans 
d'autres sphères sociales. Le petit génie musical est à l'orchestre ce que le petit génie intellectuel est à l'école ou l'entrepreneur génial à la vie économique : tous ont en commun, le plus souvent, la corrélation entre leur «talent inné » et leur origine sociale favorisée. Le discours du «talent » a ceci de mythique qu'il est fermé, car circulaire, et donc inattaquable par la pensée critique. Le talent repose en dernière instance sur un sophisme, où le postulat (A) « on a du talent» s'appuie sur le postulat (B), « la preuve en étant qu'on occupe telle position ", qui lui-même s'appuie sur le postulat (A) : «on occupe telle position parce qu'on a le talent nécessaire ».

Cela ne signifie en rien que le discours scientifique soit capable de résoudre la question de l'existence ou non du «talent». Si des corrélations fortes sont démontrées et constatées depuis longtemps entre une origine sociale et des aptitudes données, elles ne sont jamais mécaniques et absolues. Le discours du "talent ", néanmoins, ne peut être accepté par l'appréhension scientifique et conceptuelle en ceci qu'il est non critique et irréfutable autant qu'improuvable, de la même manière que la croyance en l'existence d'un dieu quelconque.

La forme moderne de l'orchestre symphonique charrie donc sa part d'archaïsme. Avec la destitution de la société d'Ancien Régime, qui se fantasmait comme figée et régie par l'ordre divin, la société bourgeoise, finalement, a promu un nouveau principe métasocial, mais qui, quant à lui, justifiait au cas par cas l'ascension et l'évolution sociales. À un principe statique, elle a substitué un principe dynamique, mais son fondement métasocial et religieux n'en est pas moins incontestable.

Encore une fois, l'orchestre est clairement le porteur de représentations et d'une idéologie qui le dépassent et traversent toute la structure sociale. Le type de discours justifiant l'ordre social dont il est le porteur peut d'ailleurs encore, parfois, se travestir en discours scientifique. Conceptualisons ainsi le discours de la soi-disant «sociologie de l'acteur rationnel ». L'acteur rationnel est celui qui obtient des résultats les plus proches possibles de ses attentes. Sa « rationalité » n'est donc en rien conceptuelle, elle est un artifice téléologique : l'acteur rationnel, c'est celui qui parvient à ses fins. Du coup, celui qui «réussit » en quoi que ce soit est toujours le plus « rationnel», et le résultat de ce discours que cette « sociologie " nous a transmis intact depuis l'âge de la chasse au mammouth, c'est bien évidemment que l'ordre social est toujours juste tel qu'il est, puisque ce sont les plus «rationnels» qui gagnent, et ceci car c'est précisément gagner qui est la mesure de la rationalité. L'acteur « rationnel » est à la "sociologie de l'acteur " ce que le " génie » est à la pratique orchestrale : l'homologie structurale est évidente (comme quoi le concept est bel et bien ce qui différencie le discours scientifique de tous les obscurantismes, dussent-ils se prétendre «modernes ».)

38 Mais après avoir opéré une rapide analyse structurale de l'orchestre symphonique, du public et avoir tenté de mettre en évidence l'importance primordiale de la structure sociale qui l'environne, nous allons à présent nous pencher sur le rituel du concert en tant que prétexte à des échanges entre le public et l'orchestre.

\section{Échanges et symétries dans le rituel du concert symphonique}

39 Ici, l'approche structuraliste des faits sociaux comme faits d'échange mus par une loi de réciprocité rencontre vite ses limites. Certes, il existe indubitablement des éléments de réciprocité dans un concert symphonique. Tout d'abord, la plupart du temps (bien que 
ce ne soit pas systématique), les auditeurs paient leur place pour assister à un tel concert. Nous pourrions ainsi formaliser ce rituel comme un échange marchand, réciproque, donc, entre des spectateurs qui paient une place et des musiciens qui leur fournissent la prestation demandée. Cependant, limiter le concert (en dehors, bien entendu, du cas des concerts gratuits) à ce simple échange marchand serait éminemment réducteur et inexact. Car le public n'a aucunement l'absolue certitude d'en « avoir pour son argent ». Nous le constatons ici, le principe de réciprocité n'a rien d'inéluctable ou de mécanique. Un mauvais concert serait une altération de cette réciprocité, et un tel cas n'a rien d'exceptionnel.

D'autres éléments d'échange existent néanmoins dans le rituel du concert entre public et orchestre. Cependant, les éléments de réciprocité qu'ils contiennent sont contrebalancés par des différenciations. Ainsi, le public prodigue des applaudissements, puis laisse le monopole de l'expression à l'orchestre (à moins d'une exécution calamiteuse qui provoquerait des huées). Encore une fois, les applaudissements finaux n'ont rien de garanti ni d'automatique.

41 Être applaudi d'un public exigeant (celui dont on est le moins sûr qu'il sera « réciproque ») est d'autant plus valorisant pour l'orchestre. Au contraire, l'orchestre qui ne donnerait pas ce que le public attend est dévalorisé. Les positions ne sont donc pas symétriques.

Nous nous rendons bien compte que les positions dans l'orchestre sont d'autant plus valorisées qu'elles sont dangereuses. Ceux-là mêmes qui seront le plus applaudis en cas de «bon" concert seront plus exposés dans le cas inverse: les positions les plus importantes dans l'orchestre sont donc éminemment réversibles. Nous avons ici une ambivalence typique du sacré.

43 C'est à ce moment de notre appréhension du rituel que l'analyse structurale montre ses limites. Elle nous laisse trop à la porte du rituel. Celui-ci, si on nous permet ici cette expression, est trop considéré « comme sur du papier à musique " par l'appréhension structuraliste. Il est porteur de tensions, de dissymétries, il peut échouer. Il ne saurait se résumer à un système explicatif où à un mode de classement inconscient de la réalité. La réversibilité des positions les plus décisives montre bien que ce « classement » est fragile et peut être remis en cause.

44 De plus, avec la figure du génie et le discours du talent, nous avons d'ores et déjà souligné la présence d'éléments dans ce rituel qui semblent bien avoir des points communs avec les phénomènes religieux et avec le sacré. Nous allons donc à présent effectuer un détour par la théorie de René Girard pour conceptualiser le sacré, afin d'en repérer la présence dans le rituel du concert et d'en comprendre éventuellement l'objet et le sens.

\section{Concert et sacralité}

\section{Girard et la problématique de la violence : qu'est-ce qu'un rituel ?}

René Girard a bousculé l'anthropologie en partant d'une constatation simple. La quasitotalité des mythes, remarque-t-il, racontent la même histoire, avec les mêmes éléments, de manière stéréotypée. Cette histoire, la voici : il s'agit du massacre, de la persécution ou de l'expulsion d'un bouc émissaire. Une crise sociale remettant en cause 
les différenciations établies (que Girard nomme " crise d'indifférenciation ») advient. Il peut s'agir d'une peste, d'une famine ou bien d'un phénomène naturel catastrophique. Or, selon les mythes, cette crise a été provoquée par un crime que Girard dit indifférenciateur, c'est-à-dire un crime qui remet en cause des différenciations indispensables à la vie en société. Citons ainsi le parricide ou l'inceste, qui remettent en cause la différence générationnelle, ou le cannibalisme, qui abolit la distance homme/ animal. Selon le mythe, le coupable de ce crime est désigné par un stigmate sacrificiel, particularité physique ou sociale qui le différencie du groupe. Le groupe, dès lors, persécute, massacre ou expulse le bouc émissaire, qui est ensuite, par un retournement qui semble à première vue incompréhensible, sacralisé comme garant des normes du groupe.

Ce que comprend Girard, c'est que les mythes racontent une histoire en inversant les rapports logiques liant crise sociale et individu. L'événement réel que racontent les mythes, selon Girard, se déroule ainsi : une crise sociale provoque l'advenue de la violence, phénomène mimétique. En effet, lorsque les cadres sociaux fonctionnent normalement, le désir mimétique, désir d'un objet par l'imitation d'un rival, est conjuré la plupart du temps. L'interdit de l'inceste empêche que père et fils, ou mère et fille, désirent la même personne (la mère dans le premier cas, le père dans le second); les systèmes d'échanges matrimoniaux ou symboliques ont le même effet: empêcher la lutte pour la possession de la même chose, personne ou bien.

En période de crise, les rapports peuvent néanmoins dégénérer vers la violence mimétique. La volonté de posséder un bien au détriment d'un autre membre de la collectivité pousse à employer la violence. Or cette violence amène sempiternellement une réponse, elle est mimétique et peut entraîner un cycle ininterrompu de vengeances qui peuvent mener à l'anéantissement de la société. La désignation d'un membre marginal de la collectivité, voire d'un étranger, permet de conjurer ce risque de violence de tous contre tous pour la canaliser vers une victime unique, ou un petit groupe sans défense.

Ainsi, lors de la crise sacrificielle, une victime est désignée par les persécuteurs selon des signes victimaires, afin de faire converger sur elle la violence de tous, ce qui va recréer l'unité du groupe. Ces signes permettent en fait de choisir une victime minoritaire ou marginale, ce qui assure que la réciprocité de la violence mimétique sera brisée : la victime ne pourra pas se défendre et personne ne la vengera. Mais pour être efficace, cette projection collective doit être inconsciente ${ }^{2}$. On va donc accuser la victime d'un crime "indifférenciateur", crime dont la contagion supposée dans le corps social est fantasmée comme la cause réelle de la crise sacrificielle. Une fois désignée, la victime est sacrifiée ou expulsée par le groupe : elle est ensuite sacralisée car son sacrifice permet d'instaurer ou de restaurer l'ordre culturel. En effet, le massacre du bouc émissaire permet à la collectivité de retrouver une unité que la violence mimétique avait mise en danger, unité réalisée aux dépens de la victime. Puisque la mort ou l'expulsion de celle-ci met fin à la violence mimétique, c'est, pour ses persécuteurs, la preuve qu'elle était la cause de la crise.

Le rite sera enfin, selon Girard, le moyen par lequel la société rejouera la scène originelle de massacre, sous une forme euphémisée, pour en retrouver les vertus pacificatrices. La victime originelle sera, au fur et à mesure que les différenciations seront établies et la violence symbolisée, remplacée par une victime de substitution, 
animal sacrificiel voire carrément objet inanimé. Le lynchage du départ sera remplacé par une scène de plus en plus métaphorique.

Le rituel est donc selon Girard la reprise d'une scène pour lui originelle, dont il reprend les éléments salvateurs afin de protéger la société de sa propre violence, par le biais de l'établissement d'une différenciation fondamentale, celle entre le bouc émissaire et les autres, entre le sacré et le profane. Dès lors, le sacré cesse d'être, comme pour les structuralistes, une sorte de monstruosité ou de paradoxe incompréhensible. Son ambivalence est la conséquence de la violence qu'il contient: violence qu'il faut éloigner de la société pour la sauvegarder, mais qui, sacralisée, lui revient sous une forme civilisatrice, en établissant des différenciations qui conjurent les rivalités mimétiques.

51 L'appréhension girardienne est très intéressante et stimulante, elle a l'avantage de fournir des concepts (bouc émissaire, mimétisme) opératoires et de donner une explication à la permanence, à travers de multiples contextes ethnographiques, de la division sacré/profane, permanence que le structuralisme n'explique guère. Cependant, la théorie girardienne a elle aussi ses limites, que nous ne pouvons énoncer ici dans leur totalité. Néanmoins, nous allons en souligner certaines.

Ainsi, pour Girard, l'aspect stéréotypé des mythes ne peut signifier qu'une chose : ils se rapportent à une scène originelle réelle. Toutes les sociétés humaines seraient issues du massacre d'un bouc émissaire qui aurait marqué le début de l'ordre symbolique et social. Cependant la description girardienne de cette scène contient un cercle. C'est pour conjurer le désir mimétique que la foule persécutrice massacrerait le bouc émissaire, nous explique Girard. Mais pour désirer quelque chose, il faut déjà être fait de symboles, autrement dit, il faut déjà que l'ordre symbolique, dont sa théorie prétend élucider l'origine, existe. De même, pour désigner le bouc émissaire comme différent du reste du groupe, il faut déjà que des différenciations existent, par définition.

De plus, bien des récits contenant les éléments girardiens du mythe ne sauraient se rapporter à un événement réel, ce qui remet en cause sa théorie. Dans la chanson L'hécatombe de Georges Brassens, des ménagères « se crêpent le chignon » «à propos de bottes d'oignons" (crise d'indifférenciation mettant en jeu la rivalité mimétique), et retournent leur violence contre les gendarmes, car « dès qu'il s'agit de rosser les cognes, tout le monde se réconcilie» (projection de la violence du groupe sur un groupe qui ne peut la renvoyer). Ces gendarmes sont d'ailleurs porteurs d'un stigmate sacrificiel : les ménagères « leur auraient même coupé les choses ", mais "par bonheur ils n'en avaient pas ». On trouvera même le crime indifférenciateur dans la chanson avec le "vieux maréchal des logis » à qui l'on fait crier "mort aux vaches, mort aux lois, vive l'anarchie » (voilà qui remet en cause les différenciations établies!) Or, nous conviendrons tous que des fouilles archéologiques sur la place du «marché de Brive-laGaillarde » échoueraient sans nul doute à nous apporter la preuve de la véracité de la scène!

Autre faiblesse, la théorie girardienne du mimétisme repose sur un glissement sémantique du terme qui recoupe au final des réalités très différentes. Le désir mimétique est d'abord fruit de l'imitation d'un rival, puis cette imitation crée la rivalité, cette rivalité crée le mimétisme de la violence («œil pour œil, dent pour dent »). Nous constatons la différence ici entre un « mimétisme d'apprentissage » et un «mimétisme de la violence ». Or, il est tout à fait possible d'avoir l'un sans l'autre. Je peux très bien lire La Princesse de Clèves parce qu'on me l'a conseillé et ne pas vouloir 
entrer en rivalité avec un autre de ses lecteurs. À l'inverse, quant à la suite d'un courant d'air, une porte claque et m'arrive dans la figure, je peux très bien, dans un accès de violence mimétique, lui flanquer un coup de pied rageur : à quel moment a-telle été pour moi rivale ou modèle? Il y a chez Girard une critique post-soixantehuitarde et un brin réactionnaire du désir, qui ne serait qu'acte d'imitation et négation de l'authentique liberté. Or le désir contient aussi une potentialité de dépassement de soi-même, de création de formes nouvelles.

Nous ne sommes donc pas plus " girardien orthodoxe » que "structuraliste béat », mais la théorie girardienne a au moins le mérite de nous fournir les bases pour une définition du rituel, afin de l'appliquer au concert symphonique. Nous proposons donc de définir comme rituel une pratique collectivement instituée (même si elle se déroule dans la solitude, comme certaines prières par exemple), répétée, qui a pour objet d'affirmer l'existence d'une différenciation qui garantit l'ordre du groupe en conjurant les rivalités. Cette définition nous permet d'englober de nombreuses pratiques, qu'il est possible et nécessaire de différencier ensuite.

Nous pourrions appliquer cette définition à une pratique que nous avons pu observer chez nos grands-parents, originaires du pays d'Auge en Normandie, celle de la découpe $\mathrm{du}$ poulet lors du repas familial. C'est le père (détenteur de la violence légitime) qui a le monopole de la découpe du poulet (acte dont nous pouvons appréhender la familiarité avec le sacrifice), il conjure les rivalités en distribuant les morceaux de l'animal, selon une norme collectivement instituée qui établit des différenciations. Dans ce cas, le blanc est traditionnellement donné aux enfants, les cuisses au père et le croupion à la mère. Nous avons ici tous les éléments du rituel tel que nous l'avons défini.

Mais quel est donc l'objet du rituel, au-delà de la conjuration de la violence ? Durkheim voyait en lui un moment d'effervescence sociale, c'est-à-dire un temps où le groupe se manifeste à lui-même comme réalité agissante. L'efficacité du rituel, selon Durkheim (et Girard ne le contredit pas) n'est qu'incidemment matérielle (bien des rites ayant pour but, par exemple, de faire pleuvoir, n'ont aucune efficacité réelle sur le phénomène qu'ils prétendent modifier) : elle est avant tout sociale. Le rituel réaffirme le groupe vis-à-vis de lui-même, ressoudant ses liens et conjurant ses angoisses. Il possède une vertu cathartique et morphologique (celle de renforcer la communauté). Nous allons à présent appréhender le rituel du concert symphonique à travers ce prisme.

\section{De la sacralité dans le concert ?}

Nous l'avons constaté plus haut, le concert symphonique contient des non-réciprocités fondamentales. Il s'articule autour de dissymétries indispensables à l'existence d'un univers de sens. Ainsi, avons-nous souligné, l'orchestre monopolise-t-il durant le concert l'expression sonore, exigeant le silence. Les éventuels applaudissements n'arrivent qu'ensuite, ils sont différés. Il ne saurait y avoir rivalité pour l'expression entre public et orchestre. Cette rivalité, elle peut apparaître précisément quand le rituel échoue : des huées se font entendre pendant l'exécution si le public renie la légitimité du monopole de l'orchestre, s'il trouve son exécution mauvaise ou insatisfaisante. Nous pouvons remarquer que, dans ce cas critique, la réciprocité devient plus directe, dégénérant vers la rivalité et la violence. 
59 La prégnance du schème qui oppose, comme nous l'avons fait remarquer, l'unique au multiple semble bien abonder dans le sens d'une interprétation girardienne du concert. Les positions les plus prestigieuses, uniques, sont les plus différenciées mais aussi les plus ambivalentes. En cas de "bon" concert, bien reçu par le public, le chef d'orchestre, les solistes ou le premier violon seront les premiers bénéficiaires des applaudissements et des félicitations. Mais dans le cas contraire, ils seront les plus exposés. Leur statut a bien des points communs avec celui du bouc émissaire : ils sont, plus que le reste de l'orchestre, mis en demeure de fournir au groupe, au public, ce qu'il vient chercher. En cas de succès, ils obtiennent un statut exceptionnel et valorisé, nous pouvons dire sans exagération qu'ils sont sacralisés. Mais dans le cas contraire, c'est la scène que Girard pense matricielle qui s'offrira au regard de l'observateur: huées, sifflements, insultes deviendront le substitut euphémisé du massacre. Dans le positif comme dans le négatif, le groupe envoie quelque chose qui ne revient pas directement vers lui, violence ou admiration. Il est évident que l'extériorité ainsi marquée est au cœur du sens même du rituel.

60 N'importe qui ne peut pas monter sur l'espace différencié, sacré, de la scène. L'orchestre est conjuré le temps du rituel, avant d'être remis à distance, loin de la banalité du quotidien, à la fin de ce temps. La scène du concert a bien des affinités structurales avec le baku, la place centrale du village trobriandais. Encore une fois, si le concert symphonique est une forme moderne, sa structure n'échappe nullement aux fondamentaux anthropologiques et contient bien des éléments typiquement archaïques. Si le «talent» des musiciens ou du compositeur n'est pas aussi incontestable pour l'auditeur que la loi traditionnelle des ancêtres sacrés des trobriandais l'était pour eux, il a comme cette dernière un fondement qui est souvent pensé comme métasocial, extérieur au groupe. La forme symbolique du concert nous pousse donc, non à nier, mais à questionner avec prudence la différence entre modernité et archaïsme ou tradition qui est une des tartes à la crème des sciences humaines. La liberté et la capacité collective et individuelle à la création de formes nouvelles ne peuvent s'affranchir de l'obéissance aux fondamentaux, à l'« alphabet » de la condition humaine.

61 Les positions les plus marquées, dans l'orchestre comme dans le public, sont des positions de médiation, dans le sens qu'elles relient des sphères différentes tout en marquant leur extériorité l'une vis-à-vis de l'autre. Le chef d'orchestre est au croisement de sphères multiples, et sa position est la plus prestigieuse autant que la plus dangereuse : c'est un passeur entre le compositeur et l'orchestre, entre ce dernier et le public. Mais le passage qu'il autorise d'une sphère à l'autre n'est aucunement abolition de la distance entre elles. C'est lui qui impose une exécution au musicien, pas l'inverse. C'est lui qui propose sa vision au public, qui l'emmène vers l'œuvre. De la même manière, le premier violon est le lien entre les violons et le chef, mais il est là encore le vecteur d'une circulation qui n'est par essence pas réciproque : il retransmet la vision du chef, qui retransmet celle du compositeur et pas l'inverse.

Des figures homologues sont identifiables dans le public, du spectateur habitué et sûr de son goût qui sera à l'origine d'applaudissements ou de huées que bien d'autres suivront (et qui introduit ainsi le néophyte dans l'espace du rituel), pont entre public et orchestre, au critique dont on attendra le jugement avisé avant de former son jugement sur l'œuvre, car lui possède un contact avec le « ciel » de la légitimité (lui et pas nous, encore une fois). 

familiarité que ses différences avec un match de football, rituel durant lequel la distanciation n'est pas marquée de la même façon), réaffirme la nécessité pour un groupe humain de s'appuyer sur un « au nom de » qui soit un minimum distancié, posé en dehors de lui-même. Certes, ce n'est certainement pas l'apprenti anthropologue que nous sommes qui reprendra à son compte le discours du croyant, selon lequel ce qui est sacré est extérieur et indiscutable. L'apport de la modernité est bien, en ce domaine, la possibilité de discuter, de critiquer, d'objectiver les choses les plus sacrées. Mais cette discussion elle-même se fait au nom de quelque chose. De la même façon qu'au moins au moment où j'assiste à un concert symphonique, j'accorde par là même aux musiciens un minimum d'exceptionnalité, au moment où je me livre à la pensée critique, je prête, par définition sans preuve, à cette même pensée le fait de valoir le coup de s'y livrer. Il y a là le point de contact, nous semble-t-il, entre art, religion, politique et science : ces activités reposent toutes en dernière instance sur un acte de croyance, même si leurs démarches respectives ne prêtent pas le même statut à la croyance.

$\mathrm{Au}$ cœur du discours artistique de la modernité, qui en appelle au "génie» révolutionnaire et créatif contre la reproduction aliénante de la tradition et du déjà fait, nous pouvons, non sans une certaine ironie, retrouver les traces du discours le plus « traditionnel » et le plus archaïque, celui du bouc émissaire, stigmatisé, massacré puis sacralisé. Dans la musique et dans d'autres domaines artistiques, de Mozart le génie mort tragiquement à Van Gogh qui se coupe l'oreille, de Baudelaire puni par la censure avant d'être porté au pinacle du panthéon culturel français à Rimbaud le poète maudit homosexuel, que de sacrifices, de stigmates, de crimes indifférenciateurs, d'expulsions se retournant en sacralisations! Tout cela est-il bien moderne?

Ces affirmations nous placent dans une certaine distance critique, nous ne le cacherons pas, vis-à-vis des pensées qui ne voient dans la sacralité qu'un simple masque de la domination qu'elle aurait pour objet de justifier; et plus largement dans le projet de la pensée un simple projet critique, corrosif vis-à-vis de toutes les normes, qui ne cacheraient que de l'asservissement et de l'aliénation. C'est notre point d'achoppement avec la sociologie bourdieusienne, par exemple. Alors que Bourdieu a, plus que tout autre sociologue français, compris les apports de l'anthropologie structurale pour l'étude de faits sociaux de notre propre société, sa conception du sacré débouche selon nous sur une impasse. Bourdieu, tout d'abord, n'emploie que rarement le terme de sacré, qui n'est pas franchement sa religion, c'est le moins qu'on puisse dire. Chez lui, le sacré en tant que réceptacle de la mauvaise violence mimétique pour la transformer en violence civilisatrice, en violence légitime (pour reprendre grosso modo la définition girardienne) est rebaptisé « légitimité ». Or, la théorie de la légitimité de Bourdieu, et la théorie de la «violence symbolique » qui en est le corollaire sont profondément boiteuses.

66 En effet, pour Bourdieu, toute légitimité n'est que le résultat, la superstructure en quelque sorte, d'un rapport de force social qu'elle retranscrit et légitime. La légitimité n'est donc que le masque de la violence, de la violence symbolique, violence composée de signes qui impose la domination à celui qui subit la légitimité. L'orchestre fait ainsi subir la violence symbolique au public en le forçant à se taire.

67 Cependant, le langage, ou n'importe quel acte porteur de symboles, fait subir à l'acteur la violence de s'y conformer. Est-on pour autant plus libre de la domination en refusant ces actes et, partant, la société ? Nous tombons ici dans une aporie. Soit la légitimité est

Appareil, 3 | 2009 
issue de la domination, et comme toute société nécessite un partage entre chose légitime et illégitime, alors il serait impossible de faire société sans domination (ce qui est difficile à avaler de la part d'une sociologie qui entend critiquer la domination!) Notons immédiatement que ce premier point de vue est indéfendable : il a existé bien des sociétés où les rapports hiérarchiques entre classes n'ont pas été observés et dans lesquelles il existe des choses sacrées (légitimes, si vous y tenez). Soit il peut donc exister des légitimités qui ne découlent pas de la domination ni n'ont pour objet de la défendre (et c'est notre point de vue) et la sociologie critique de la "violence symbolique " se clôt sur un échec théorique. Nous sommes après tout en droit de trouver un peu gênant de construire une sociologie qui bute sur la "simple" impossibilité à rendre compte de ce qu'est une société. Ce ne sont certainement pas les tenants d'une sociologie critique qui nous reprocheront de les critiquer!

Certaines positions de dissymétrie symbolique sont protectrices et circulantes (en effet, le «sociologue critique » qui « dévoile » la domination impose sa violence symbolique, mais laisse à celui qu'il a "éclairé » sa place symbolique par la suite, et on ne lui en voudra guère) et d'autres sont créatrices de domination (se moquer des manières d'une personne parce qu'elles font "populaire ", c'est de la violence symbolique, mais est-ce vraiment exactement la même chose que de donner un cours de sociologie? Et si oui, alors quel est donc le problème que pourrait poser la domination, puisque tout ce qui est symbole serait violent ?) et non circulantes. Empêcher un enfant de taper un plus faible dans une cour d'école, c'est lui imposer une violence symbolique. Mais y a-t-il une société dénuée de domination possible sans cette violence?

Nous avons essayé de le démontrer, le relativisme, pour ne pas dire le plat nihilisme, dans lequel se fourvoie la part idéologique de la sociologie bourdieusienne s'écroule assez vite sur lui-même et n'apporte rien de bien libérateur, si ce n'est une sorte de cynisme pseudo-aristocratique («toute valeur est critiquable donc fichons-nous de tout ») qui correspond assez bien à l'idée que les bourdieusiens se font du bourgeois décadent.

Le rituel, loin de n'être qu'un acte d'aliénation, est une façon pour le groupe de se poser en extériorité vis-à-vis de lui-même, de se confronter à (et de matérialiser) son image idéale. Le rituel du concert symphonique est, selon nous, un de ces temps ou le groupe met en demeure une de ses parties qu'il met à distance de lui-même de lui procurer une extériorité dont il est en fait le créateur et dont il a besoin pour s'instituer et se confirmer comme réalité agissante. Si l'injonction est couronnée de succès, les artistes sont sacralisés, tenus dans une distance respectueuse, mais tout autant prêts à être régulièrement convoqués et conjurés pour faire revivre ce nécessaire instant de grâce. L'échec, quant à lui, renvoie la sphère sacrée à son objet archaïque: devenir le réceptacle d'une violence qui anime le corps social et dont il se débarrasse à cette occasion, dans une pratique toute cathartique.

\section{Le concert symphonique et la problématique de l'indifférenciation}

71 Nous pensons donc en avoir offert plus d'un élément de démonstration, le rituel du concert symphonique, en tant qu'élément d'un système symbolique plus vaste, ne saurait être appréhendé comme une réalité autonome vis-à-vis du contexte social et culturel qui l'entoure. Des homologies peuvent et doivent être constatées entre ce qui 
se produit dans le «microcosme » du rituel et dans le "macrocosme» du «tout» social. Nous allons donc tenter d'apporter des éléments d'analyse de phénomènes esthétiques tels que le rituel qui nous intéresse ici pour les mettre en relation avec des problématiques politiques qui le dépassent et lui sont en apparence étrangères, par le jeu de la comparaison de structures communes, de la mise en évidence d'homologies structurales.

Les discours politiques, en France mais aussi dans d'autres pays industrialisés modernes, contiennent des éléments d'indifférenciation facilement appréhendables. Prenons par exemple le slogan de Ségolène Royal, lors de l'élection présidentielle de 2007 : «La France présidente ». Dans ce slogan, la distanciation entre le peuple et son représentant est symboliquement abolie, dans un schème fusionnel. Ce schème fusionnel nous renvoie assez vite à l'archaïsme du bouc émissaire : celui qui serait en désaccord avec le ou la présidente ne serait plus membre de "la France», puisque ce serait directement elle qui serait "présidente». Nous ne sommes plus ici dans la représentation, mais dans l'incarnation. Bien des distanciations et des différenciations sont destituées au passage, et nous pensons être fondés à voir là des symptômes de crise. La même candidate, durant la même campagne, en appelait à ce que chaque Français ait le drapeau national chez lui, proclamation qui, là encore, anéantit des différenciations fondamentales et contient un problème vis-à-vis de la sacralité. Espace intime et espace privé ne sont plus distanciés (la puissance publique pouvant fantasmatiquement exiger la présence du drapeau dans l'espace intime), ceci en homologie (inconsciente mais d'autant plus révélatrice) avec une pratique politique qui place l'intimité de la candidate (nous avons pu constater la même chose chez le candidat concurrent, Nicolas Sarkozy) sur le devant de la campagne politique. On aura alors vu des photographies de ladite candidate en maillot de bain, ou l'autre candidat exhiber sa vie de famille comme argument électoral. Chez Nicolas Sarkozy, la thématique de l'indifférenciation est aussi primordiale. Son slogan «ensemble tout devient possible » renvoie au religieux le plus archaïque, celui qui pense que le symbole est matériellement agissant, qui se paie de mots. La différenciation entre mot et chose, entre matière et symbole, entre réalité et fantasme, s'efface.

Or, nous pensons pouvoir relever des traces de cette indifférenciation dans d'autres dimensions de la pratique sociale. Citons par exemple le cas d'une publicité mettant en scène un match de tennis. Peu à peu, les membres du public entrent sur le terrain pour jouer aux côtés des tennismen, et le film se termine par une scène très girardienne de crise d'indifférenciation, ou tout le monde se retrouve sur le terrain. À travers cet exemple, nous constatons bien la prégnance d'une crise de la distanciation et de la différenciation. Le fait que le joueur professionnel se distingue et se distancie du spectateur moyen devient inacceptable. Mais si tout le monde devient joueur de tennis, sans distinction de niveau de jeu, alors le rituel du spectacle du match disparaît, faute de public, et n'a plus aucun sens. S'il n'y a plus de bons et de mauvais joueurs, les premiers pouvant servir de modèles aux seconds, alors la pratique du tennis n'a plus aucun sens ni aucun objet. On ne peut par définition se réaliser et se révéler à soi-même dans une activité qui ne pourrait être «bien » ou « mal » effectuée.

Ces schèmes indifférenciateurs, nous pouvons les retrouver dans le champ de la musique.

Prenons tout d'abord l'exemple des exécutions de La Marseillaise sifflées à l'occasion de matches internationaux de football. La définition de la sacralité que nous avons fournie 
s'applique ici sans contestation possible: l'hymne est vénéré, marqué de tabous et d'obligations, tenu à distance (interprété par des musiciens au centre du stade) mais conjuré (tout le public le reprend en chœur), enfin ambivalent (certains le sifflent). Siffler La Marseillaise est à présent passible de prison et les retransmissions télévisées, lors de l'hymne, offrent au spectateur la possibilité de vérifier, à l'aide d'une caméra et d'un micro, si les joueurs chantent bien l'hymne national. Il y a clairement là une modification du régime du symbole et de la sacralité. Là encore, l'hymne n'est plus représentation mais incarnation du peuple français. La critique devient acte de profanation : ceux qui, originaires de pays colonisés par la France et placés en situation d'infériorité et de stigmatisation sociales, sifflent cet hymne sont symboliquement expulsés du corps social, pensé comme unanime et fusionnel. Or, l'appréhension anthropologique nous montre bien que ces siffleurs ne sont pas des «sauvages » qui ne respectent rien. C'est précisément parce qu'ils comprennent et reconnaissent la sacralité de l'hymne qu'ils le sifflent! L'exécution dans le même contexte du Petit Vin blanc ne provoquerait sans doute pas les mêmes réactions... Si nous définissons la modernité politique comme l'advenue de la possibilité de porter un regard critique, de discuter ce qui est sacré, alors la loi «anti-sifflets» ne relève clairement pas de la modernité politique. Mais les choses sont encore plus complexes à notre avis : cet exemple montre que la modernité ne peut s'abstraire de l'archaïque. Nier qu'il est indispensable que des choses sacrées existent pour qu'il existe une collectivité politique peut provoquer en retour une réaction hystérique vis-à-vis de la sacralité.

Autre exemple dans le champ de la musique : les concerts d'André Rieu. André Rieu est un musicien classique qui a décidé de jouer pour un public qui n'est pas celui du concert symphonique. Ce public, vu le prix élevé des places d'un concert de Rieu, nous pouvons penser qu'il est pourvu de ressources économiques certaines, mais, nous allons le constater, qu'il n'est pas introduit aux codes esthétiques de la musique et du concert classiques. D'ores et déjà, nous pouvons constater que Rieu tient un rôle de médiation typique du sacré : il apporte la " grande » musique à un public exclu du rituel du concert symphonique. Mais le visionnage de ses concerts nous permet de repérer le schème fusionnel et indifférenciateur que nous avons relevé dans différentes sphères de l'activité sociale. Rieu, lors de certaines exécutions, mime de manière caricaturale le soliste classique inspiré, provoquant le rire du public qui chante la mélodie en chœur. Nous avons ici un phénomène fusionnel: l'orchestre n'a plus le monopole de l'expression sonore, au moment précis où la figure sacralisée est destituée, dans un mode qui semble avoir des familiarités avec le carnavalesque. Il y a cependant bien une distanciation au sens girardien: on communie dans la détestation de l'artiste prétentieux représenté par Rieu, sur lequel on projette la violence du groupe, à distance, donc. L'indifférenciation plus forte que lors du rituel symphonique classique (où l'orchestre joue et le public est silencieux) conduit à une scène qui a une familiarité certaine avec la scène girardienne de massacre du bouc émissaire. C'est un groupe social démuni des codes culturels légitimes qui exerce dans ce rituel sa catharsis en projetant dans un mode encore une fois non réciproque sa violence sur un représentant de la légitimité culturelle honnie. Cependant, nous devons nous interroger sur l'efficacité de cette catharsis du ressentiment. Quel ordre culturel institue-t-elle, quelles différenciations et quelles valeurs? En quoi communier dans la haine de la culture instituée permet-il d'affirmer des normes définissant positivement le groupe ? Ce rituel archaïque en pleine modernité ne contient-il pas la clé d'une appréhension anthropologique de phénomènes politiques tels que le fascisme? 

porte à un comparatisme qui ne mène pas selon nous au relativisme. Certains rituels permettent l'affirmation de distanciations et de différenciations plus marquées et plus instituantes que d'autres. Si le concert symphonique et le concert de Rieu sont tous deux des variations autour des éléments archaïques du rituel, leurs résultats diffèrent. Le concert symphonique s'éloigne visiblement du religieux archaïque, de la projection de la violence du groupe, il la sublime davantage et institue des différenciations protectrices. Le concert de Rieu est beaucoup plus fusionnel, marqué par des réciprocités plus directes, il ne sublime pas de la même manière la violence du groupe qu'il projette, mais ne fait pas revenir vers lui pour instituer un être-ensemble. Il nous semble qu'il est possible d'identifier des régimes différents de la sacralité, et que c'est dans la variété de ces régimes que se pose la question de la différence entre l'archaïsme et la modernité. Le concert symphonique marque, malgré certaines limites, un rapport beaucoup plus critique et distancié vis-à-vis de la sacralité que le concert «à la Rieu ", fusionnel, violent, fondant le groupe sur l'expulsion de tout ce qui diffère dans l'adoration sans distance du chef.

Politiquement, la problématique de la sacralité et de la différenciation nous semble bien posée par la fin du film Le Goût des autres. Nous y voyons un personnage jouer à la flûte traversière un morceau simpliste, mais qui lui a demandé les plus grands efforts. Cette fin valorise la capacité de chaque être humain à se dépasser vers le beau, mais elle contient, sous peine de devenir aporétique, la réaffirmation des différenciations et des hiérarchies de valeurs. Il y a une différence primordiale à nos yeux entre le projet humaniste qui affirme la possibilité pour chaque être humain d'accéder à son propre dépassement, dépassement qui n'est possible que dans un monde différencié et porteur de valeurs instituées; et le projet répressif, corrosif et tyrannique d'égalité dans la détestation, l'indifférenciation et la médiocrité. C'est pour nous la frontière entre démocratie et totalitarisme. La démocratie ne peut, ne doit s'abstraire de fondements sacrés, mais la sacralité démocratique est distanciée, critiquable, négociable, pensée comme le résultat d'une œuvre humaine et non d'un au-delà du social. En d'autres termes, le rituel du concert symphonique nous pousse à poser la frontière entre moderne et archaïque, non entre Nous et les Autres cultures, mais au sein même de notre réalité politique et sociale, voire au sein même de notre propre expérience de vie.

\section{Pensée critique, archaïsme et modernité ${ }^{3}$}

Nous avons donc tenté d'offrir un état des lieux, bien entendu vite limité par notre compétence, de ce que l'anthropologie du sacré pouvait apporter à un regard conceptuel sur le concert symphonique, et à travers lui, sur les phénomènes culturels et esthétiques formalisés en tant que pratiques rituelles.

Sans renier les apports considérables du structuralisme, nous avons essayé d'apporter la démonstration que ce courant théorique avait refermé à tort la question durkheimienne du sacré. Une société n'est pas qu'un système de classement inconscient. Elle est structurée par des logiques, des forces découlant de sa morphologie et de son fonctionnement. Elle doit opérer sur elle-même un travail permanent et qui ne réussit toujours qu'en partie pour canaliser et sublimer les forces qui découlent de son inaptitude fondamentale à être fidèle à son image idéale. Une société a besoin d'une sphère séparée d'elle-même, même si elle en est l'origine, afin de 
déverser sa violence, ses angoisses, ses contradictions et ses espoirs afin non seulement de s'en purger, mais aussi de les voir revenir à elle transformés en formes instituées. C'est pourquoi le sacré est toujours en contact et à distance : parce qu'il differe. Le rituel ne répond pas qu'à des impératifs de logique ou de classement. Il est une sorte de praxis sociale où la société accouche d'elle-même, en donnant forme à ses angoisses informes et en se réaffirmant à elle-même comme réalité sensible et agissante.

Dès lors, la question du sacré ne saurait se résoudre à celle de l'aliénation, ou la question de la légitimité à celle de la domination. Si le sacré peut être dangereux, véhicule de l'obscurantisme et de la violence les plus aveugles, il a aussi sa raison d'être. S'il est l'opium du peuple, il l'est aussi bien en tant que poison qu'en tant que calmant qui a pu être utilisé pour la chirurgie. Il répond à des impératifs fondamentaux. Nous avons essayé de le démontrer, on ne peut critiquer avec honnêteté intellectuelle et cohérence relative la domination qu'au nom de choses qu'on trouve légitimes et qui ne la justifient pas. Si la société pose une part d'elle-même à distance, c'est parce qu'elle a quelque chose à envoyer ailleurs pour que cela puisse lui revenir transformé.

Cet impératif anthropologique, archaïque, ne clôt certainement pas pour nous les questions de la liberté et de la politique, mais les ouvre d'une manière qui, nous l'espérons, est plus lucide. La modernité, défendons-nous en substance, ne s'abstrait pas de l'archaïque mais le comprend et le dépasse.

Il y a donc une familiarité qui nous semble peu contestable entre les phénomènes que le sens commun qualifie de religieux, politiques ou artistiques. Ils correspondent selon nous à des régimes différents de la même réalité sociale, celle de la division entre choses sacrées et profanes qui institue un monde de sens et la cohésion du groupe. De même, il y a une structure commune entre le rituel moderne du concert symphonique et les rituels des sociétés sauvages ou traditionnelles.

Cependant, malgré la ressemblance structurelle, des différences extrêmement importantes doivent être constatées. Le rituel moderne du concert symphonique n'est pas accompagné du sentiment d'éternité immuable, de la croyance absolue qu'il aurait été donné au groupe par un au-delà de lui-même, du voile qui empêcherait toute discussion sur sa légitimité. Bien que toujours largement mus par des schèmes, par des impératifs inconscients, les hommes qui l'ont créé, qui le présentent et y assistent, ont conscience qu'il a été transmis et créé par d'autres hommes, qu'ils peuvent avoir un rôle dans son évolution et sa transformation. Certes, certains peuvent toujours croire en une présence méta-sociale en lui, par la médiation du " génie », de l'instrumentiste ou du chef d'orchestre "talentueux ». Le regard scientifique ne peut d'ailleurs, à notre avis, en rien contredire définitivement cette croyance.

Mais, dans un monde de sens où l'appréhension scientifique a, pour l'essentiel, fait le deuil de la plénitude du monde, compris plus que jamais que « tout ce qu'elle sait c'est qu'elle ne sait rien ", la pratique artistique et, partant, musicale, est peut-être l'un des derniers havres modernes de réconciliation avec cette idée de plénitude. Qu'elle s'efface, et c'est la forme primordiale, effrayante, du bouc émissaire, de la catharsis par l'expulsion et la purification du groupe qui écrase sur nous le rocher de Sisyphe de l'archaïsme, avec son confort, sa solidité... Mais dont le propre de l'idéal démocratique est de ne jamais se satisfaire. 


\section{BIBLIOGRAPHIE}

Malinowski Bronislaw, Les Argonautes du Pacifique occidental, Paris, Gallimard (Tel), 1989.

\section{NOTES}

1. Bronislaw Malinowski, Les Argonautes du Pacifique occidental, Paris, Gallimard (Tel), 1989.

2. Par « inconsciente», nous ne signifions pas « relevant de l'inconscient tel que théorisé par Freud », mais selon une logique qui échappe aux persécuteurs et que nous tenterons d'expliciter plus loin dans cet article.

3. Cette partie de conclusion doit énormément aux critiques fécondes de Jean-Louis Déotte, que nous remercions.

\section{RÉSUMÉS}

Poser un regard anthropologique sur le concert symphonique amène à le considérer comme un rituel, à savoir comme une pratique collectivement instituée où le groupe s'affirme à lui-même. Ce rituel, l'anthropologie structurale nous a appris à l'appréhender comme la manifestation d'un système symbolique, système de classement inconscient de la réalité porté par la société où il se déroule. Mais si l'appréhension structuraliste offre des outils inestimables pour le replacer dans son contexte social, elle échoue selon nous à pénétrer son sens le plus profond, qui ne saurait se limiter à un acte de classement et d'explication du monde. Ainsi, poser la question de la sacralité dans le concert symphonique nous semble être une piste féconde afin de comprendre que ce rituel, parmi d'autres, permet à la société de projeter ses angoisses dans un en-dehors d'elle même dont elle est créatrice, afin qu'elles lui reviennent porteuses de sens. Dès lors, les modifications du statut de la sacralité dans ce rituel permettraient d'interroger des éléments de crise sociale et de représentations présents aussi, par homologie, dans le discours politique ou dans d'autres sphères de l'activité sociale. Le sacré ne serait donc pas seulement le voile de l'aliénation et de la domination : il serait au contraire indispensable à l'existence de groupes humains. Dès lors, la question de la liberté humaine et de la politique se poserait en termes de régimes plus ou moins critiques, distanciés et réflexifs de la sacralité.

\section{INDEX}

Mots-clés : concert, orchestre symphonique, rituel, sacralité, sacré, structuralisme Personnes citées : Girard (René) 
AUTEUR

RENAUD TARLET

Sociologue 